\title{
Desempenho ocupacional e satisfaçáo de indivíduos pós-acidente vascular encefálico
}

\author{
Kátia Vanessa Pinto de Meneses, Jeanine Schuabb Duarte, \\ Vanessa de Oliveira Alencar, Ana Carolina dos Santos Pereira \\ Universidade de Brasília - UnB, Brasília, DF, Brasil
}

\begin{abstract}
Resumo: Introdução: Estudos científicos evidenciam sobre as intervenções de Terapia Ocupacional com pacientes pós acidente vascular encefálico (pós-AVE), ajudando-os a retornar ao desempenho de tarefas importantes e significativas. Objetivo: Medir as mudanças na percepção de indivíduos pós-AVE sobre seu desempenho ocupacional e satisfação após intervenção de Terapia Ocupacional. Metodologia: Estudo observacional, longitudinal do tipo prospectivo, com pacientes pós-AVE, atendidos no Serviço de Terapia Ocupacional da Unidade Mista de Taguatinga, Distrito Federal. A Medida Canadense de Desempenho Ocupacional (COPM) foi aplicada em três momentos: durante a avaliação inicial (momento 1), após 3 meses (momento 2) e após 6 meses de intervenção de Terapia Ocupacional (momento 3). Resultados: A análise após 3 meses de intervenção foi realizada com 17 pacientes sendo que todos obtiveram aumento nos escores de desempenho $(\mathrm{p}=0,000)$ e apenas um apresentou queda nos escores de satisfação. A análise após 6 meses de intervenção foi realizada com 13 pacientes e todos obtiveram melhora significativa $(p=0,000)$ nos escores de desempenho e de satisfação. Conclusão: O estudo demonstrou o aumento na satisfação e no desempenho ocupacional de indivíduos pós-AVE após intervenção de Terapia Ocupacional. O estudo sugere que a intervenção de Terapia Ocupacional auxilia o processo de reabilitação do indivíduo pós-AVE, possibilitando uma melhora no seu desempenho ocupacional e em sua satisfação e, consequentemente, em sua independência.
\end{abstract}

Palavras-chave: Terapia Ocupacional, Satisfação do Paciente, Análise e Desempenho de Tarefas.

\section{Occupational performance and satisfaction of individuals after cerebral vascular accident}

\begin{abstract}
Introduction: Scientific studies show that occupational therapy interventions with patients after cerebral vascular accident (CVA) - post-stroke patients, help them resume the performance of important meaningful tasks. Objective: To measure changes in the perception of post-stroke patients on their occupational performance and satisfaction after the intervention of occupational therapy. Methodology: An observational, longitudinal, prospective study with CVA patients treated at occupational therapy service at the 'Unidade Mista de Taguatinga', Federal District, Brazil. The Canadian Occupational Performance Measure (COPM) was applied in three distinct times: during the initial assessment (time 1), after 3 months (time 2), and after 6 months (time 3) of occupational therapy intervention. Results: The analysis after 3 months of intervention was performed with 17 patients; all showed increased performance scores $(p=0.000)$, and only one patient presented decrease in satisfaction scores. The analysis after 6 months was conducted with 13 patients; all showed significant improvement $(p=0.000)$ in scores for performance and satisfaction. Conclusions: The study shows the improvement in satisfaction and occupational performance of CVA patients after occupational therapy intervention. The study suggests that occupational therapy intervention helps the rehabilitation process of post-stroke patients, enabling them to improve their occupational performance and satisfaction and, consequently, their independence.
\end{abstract}

Keywords: Occupational Therapy, Patient Satisfaction, Task Performance and Analysis.

Autor para correspondência: Kátia Vanessa Pinto de Meneses, Faculdade de Ceilândia, Universidade de Brasília, Centro Metropolitano, conjunto A, lote 01, CEP 72220-900, Brasília, DF, Brasil

Recebido em 14/5/2013; $1^{\text {a }}$ Revisão em 25/4/2014; $2^{a}$ Revisão em 08/05/2014; Aceito em 19/6/2014. 


\section{Introdução}

O acidente vascular encefálico (AVE) é a segunda causa de morte e a principal causa de incapacidade em todo o mundo (FISHER et al., 2013; LAVADOS et al., 2007). O AVE é um evento de ocorrência súbita que ocorre com déficits neurológicos temporários ou permanentes de variadas intensidades (DRUMOND, 2007). Setenta por cento a $85 \%$ dos primeiros AVEs são acompanhados por hemiplegia e, após seis meses de AVE, apenas 60\% das pessoas alcançará independência funcional em atividades simples da vida diária, tais como ir ao banheiro e andar curtas distâncias (LEGG et al., 2007; DOBKIN, 2004).

A Terapia Ocupacional é parte importante da equipe multidisciplinar que compóe o processo de reabilitação do indivíduo pós-AVE (LEGG et al., 2007; STEULTJENS et al., 2003). Para a Terapia Ocupacional, a saúde está relacionada à capacidade dos clientes de se envolverem em ocupações e em atividades que permitam uma participaçáo desejada ou necessária em casa, na escola, no trabalho, no brincar, no lazer e na comunidade, indo ao encontro da Classificação Internacional de Funcionalidade, Incapacidade e Saúde (CIF) proposta pela OMS em 2001 (DRUMOND, 2007). A atuação da Terapia Ocupacional tem como foco principal o desempenho ocupacional, entendido como o resultado de interaçôes entre a pessoa, o ambiente e a ocupação (PEDRETTI; EARLY, 2000).

Evidências da literatura mostram que intervençóes da Terapia Ocupacional com pacientes pós-AVE ajudam a manter e melhorar as habilidades nas atividades de vida diária, reduzindo as chances de deterioração (LEGG, 2003; LEGG; DRUMMOND; LANGHORNE, 2006; STEULTJENS et al., 2003; WALKER, 1999), e que prescrições de equipamentos de tecnologia assistiva favorecem a funcionalidade, bem como o retorno à participação social (STEULTJENS et al., 2003). Estudo realizado por Gosman-Hedstrom, Claesson e Blomstrand (2002) identificou que a prescrição de equipamentos assistivos apresentou alto impacto na vida diária de idosos pós-AVE.

Uma das tendências de intervençôes na Terapia Ocupacional é o uso da abordagem centrada no cliente, que segundo Law et al. (2009) é uma abordagem que adota a filosofia de respeito e parceria com as pessoas que recebem os cuidados e serviços. Essa abordagem reconhece a autonomia do cliente e a necessidade dele em realizar escolhas e tomar decisóes de acordo com suas necessidades (WRESSLE et al., 2002).
O objetivo deste trabalho foi medir as mudanças na percepção de indivíduos pós-AVE sobre seu desempenho ocupacional e satisfação após um programa de intervenção de Terapia Ocupacional.

\section{Procedimentos metodológicos}

Tipo de estudo: Estudo é observacional, longitudinal do tipo prospectivo.

De acordo com Hossne (1984), estudos observacionais são quando indivíduos da amostra não foram designados aos grupos por processo aleatório, mas já estavam classificados nos respectivos grupos, no início da pesquisa (não é experimento). Estudos longitudinais prospectivos fornecem informaçóes sobre as variaçóes globais e individuais ao longo do tempo.

Amostra: Todos os pacientes pós-AVE atendidos pelo Serviço de Terapia Ocupacional da Unidade Mista de Taguatinga - UMT no período de agosto de 2010 a julho de 2011 e que preencheram os critérios de inclusão e de exclusão.

Local: A UMT é um serviço de média complexidade da Secretaria de Saúde do Distrito Federal. Funciona em regime ambulatorial e conta com um centro de reabilitação com atendimento de Terapia Ocupacional.

Critérios de inclusão: Pacientes pós-AVE de ambos os sexos, com idade igual ou superior a 18 anos, sem restrição quanto à classe social, encaminhados para acompanhamento de Terapia Ocupacional no serviço de reabilitação da UMT, que estavam em seu primeiro atendimento de Terapia Ocupacional após o AVE e que assinaram o Termo de Consentimento Livre e Esclarecido (TCLE).

Critérios de exclusáo: Idade inferior a 18 anos; incapacidade de comunicação ou limitação para expressar suas perspectivas, desde que não fosse acompanhado por um familiar ou intérprete, que compreendesse de perto a situação do cliente; alteraçáo cognitiva que impedisse a compreensão do instrumento utilizado (avaliado por meio da Escala de Avaliação Clínica de Demência - Clinical Dementia Rating); ter realizado atendimento de Terapia Ocupacional após o AVE e não concordância em assinar o TCLE.

\subsection{Instrumentos}

- Questionário socioeconômico elaborado pelos pesquisadores contendo informaçóes sobre gênero, renda familiar, data de nascimento, estado civil, escolaridade, suporte social e data do AVE; 
- Medida Canadense de Desempenho Ocupacional (COPM).

Um dos instrumentos utilizados para identificar as ocupaçóes que os clientes querem, precisam ou esperam fazer e avaliar o desempenho desses, bem como sua satisfação no desempenho das ocupaçôes, é a Medida Canadense de Desempenho Ocupacional (COPM). A COPM é um instrumento padronizado, em formato de entrevista semiestruturada e com um sistema estruturado de pontuação, para captar mudanças percebidas no desempenho ocupacional ao longo do tempo (LAW et al., 2009). A COPM é baseada no Modelo Canadense de Desempenho Ocupacional (MCDO) e ajuda a programar a abordagem centrada no cliente. $\mathrm{O}$ foco dessa abordagem está no entendimento do cliente sobre as suas reais necessidades, orientando-o a perceber $\mathrm{o}$ seu desenvolvimento e o seu desempenho ocupacional no seu cotidiano.

A COPM foi aplicada em três momentos: o primeiro durante a avaliação inicial dos pacientes, o segundo após 3 meses de intervenção de Terapia Ocupacional e o terceiro após 6 meses de intervenção de Terapia Ocupacional ou no momento da alta do paciente (nos casos em que a alta foi dada antes dos 6 meses de intervenção).

Os participantes do estudo assinaram Termo de Consentimento Livre e Esclarecido e o projeto protocolado sob no 178/10 foi apreciado pelo Comitê de Ética em Pesquisa da Secretaria de Estado de Saúde do Distrito Federal obtendo aprovação com o Parecer no 283/2010.

\subsection{Análise estatística}

As análises estatísticas foram processadas utilizando-se o programa Statistical Package for Social Sciences (SPSS), versão 16.0. As variáveis foram analisadas descritivamente utilizando medida de tendência central (média) e de variabilidade (desviopadrão e porcentagem). Para verificar as diferenças das médias das variáveis pré e pós-intervenção de Terapia Ocupacional, foram empregados testes paramétricos e não paramétricos, conforme verificação da normalidade dos dados. O nível de significância $\mathrm{p}=0,05$ foi considerado.

\section{Resultados}

A análise após 3 meses de intervenção de Terapia Ocupacional foi realizada com 17 pacientes, sendo $8(47,0 \%)$ do sexo feminino e $9(53,9 \%)$ do sexo masculino, com idade variando entre 36 e 74 anos
$(20-39=23,5 \% ; 40-60=58,8 \% ;>60=17,6 \%) . \mathrm{O}$ tempo médio do acidente vascular encefálico foi de 15,5 meses, sendo que o mais antigo ocorreu há 31 meses e o mais recente há 6 meses. Dos pacientes com dominância do lado direito, 76,4\% apresentaram hemiparesia no mesmo lado e $23,5 \%$ no lado oposto (esquerdo). Dos pacientes com dominância esquerda, todos apresentaram hemiparesia no mesmo lado.

Os atendimentos de Terapia Ocupacional aconteceram de 1 a 2 vezes por semana, com duração de 45 minutos. O número de atendimentos semanais foi definido pelo terapeuta ocupacional da instituição levando em consideração o número de vagas disponíveis no serviço, o estágio da doença em que o paciente se encontrava, a condiçâo financeira e o suporte familiar e social dos pacientes. As intervençôes terapêuticas foram baseadas na prática centrada no cliente. Dessa forma, o objetivo das intervençôes foi alcançar melhora no desempenho das atividades listadas como mais importantes pelo cliente, e estas foram usadas tanto como meio quanto como fim no tratamento.

Em relação ao resultado de desempenho após 3 meses de intervenção (comparaçáo entre os momentos 1 e 2), foi observado que $100 \%$ dos indivíduos obtiveram aumento nos escores (Figura 1). A média dos escores finais para o item "desempenho 1 " foi de 2,8 com desvio padrão (DP) de 1,7; para o item "desempenho 2" a média foi de 5,1 com DP de 2,3, apresentando diferença estatisticamente significativa $(\mathrm{p}=0,000)$.

Em relação ao resultado de satisfação após 3 meses de intervenção (comparação entre os momentos 1 e 2), todos os pacientes apresentaram aumento nos escores, com exceção de um paciente, que apresentou queda no escore de satisfação no momento 2 (Figura 2). Esse resultado reflete o aumento de exigência desse paciente em relação ao seu desempenho, diante da percepção do potencial de melhora, ocorrido durante as sessóes de reabilitação. A média dos escores finais para o item "satisfaçáo 1" foi de 2,3 com DP de 1,4; para o item "satisfação 2" a média foi de 4,8 com DP de 2,0, apresentando diferença estatisticamente significativa $(\mathrm{p}=0,000)$.

Após 6 meses de intervenção (momento 3) o instrumento foi aplicado em 13 pacientes, pois 1 foi a óbito e três outros receberam alta no momento 2 .

Em relação ao item desempenho, após 6 meses de intervenção (comparação entre os momentos 1 e 3), foi observado que todos os participantes obtiveram melhora significativa nos escores $(\mathrm{p}=0,000)$. A média dos escores finais para o item "desempenho 3 " foi de 6,3 com DP de 1,6. Porém, quando comparados 


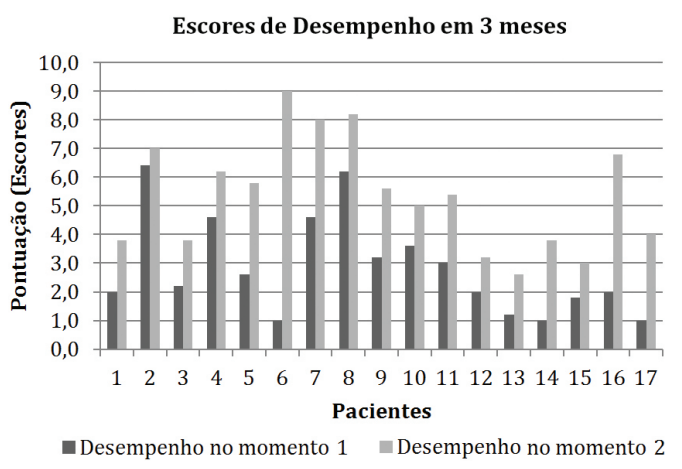

Figura 1. Escore do desempenho no momento 1 e no momento 2 .

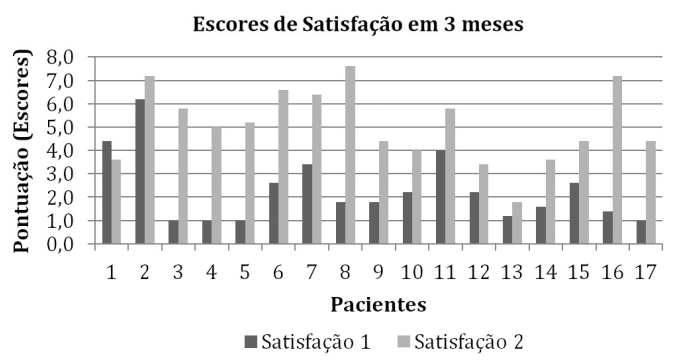

Figura 2. Escore de satisfação nos momentos 1 e 2.

os escores de desempenho de 3 meses e 6 meses de intervenção (comparação entre os momentos 2 e 3), foi observado que 11 pacientes $(84,62 \%)$ obtiveram aumento significativo $(\mathrm{p}=0,000)$ e que 2 indivíduos (15,38\%) obtiveram queda nos escores. Levando em consideração que a amostra consistiu em pacientes em diferentes estágios da patologia, e que um dos fatores que interferem no potencial de recuperação após o AVE é o tempo de ocorrência deste, uma hipótese é que a queda do escore pode estar indicando que o paciente atingiu seu potencial de recuperaçáo. A Figura 3 apresenta os escores de desempenho após 6 meses de intervenção.

Em relação ao item satisfação após 6 meses de intervenção (comparação entre os momentos $1 \mathrm{e}$ 3), houve um aumento significativo em todos os escores $(p=0,000)$. A média dos escores finais para o item "satisfação 3" foi de 5,8 com DP de 1,7. Quando comparados os escores de 3 meses e 6 meses de intervenção (comparação entre os momentos 2 e 3), foi observado aumento no escore de satisfaçáo em 10 pacientes $(76,93 \%)$ e queda em 3 pacientes (23,07\%) (Figura 4). O aumento dos escores de satisfação pode representar maior aceitação dos pacientes de suas limitaçôes e a percepção do potencial de melhora ao desempenhar as atividades que antes eram consideradas não realizáveis. A queda nos escores de satisfação pode ter sido influenciada

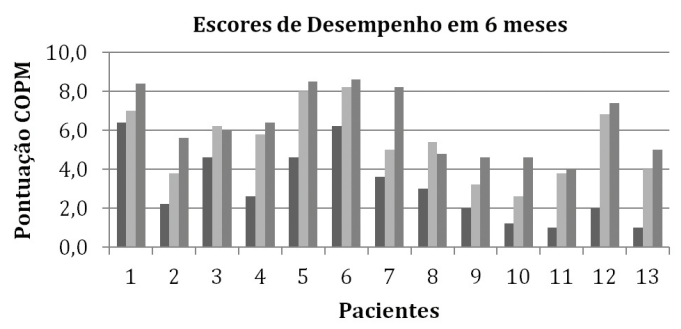

- Desempenho no momento 1 aesempenho no momento 2 - Desempenho no momento 3

Figura 3. Escore de desempenho nos momentos 1, 2 e 3 .

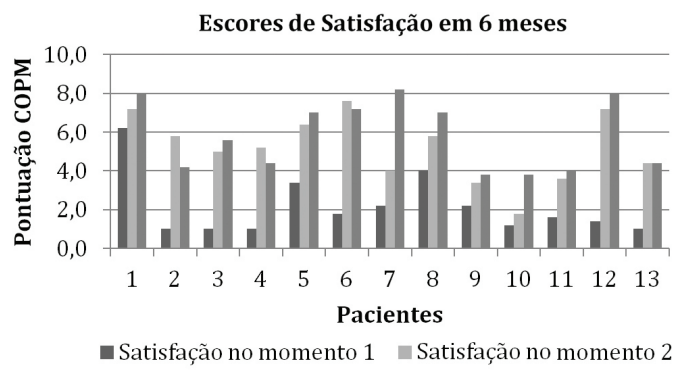

Figura 4. Escore de satisfação nos momentos 1, 2 e 3.

pelo aumento do grau de exigência dos pacientes ao realizarem as atividades.

\section{Discussão}

Neste estudo foram medidas as mudanças na percepção de pacientes pós-AVE sobre seu desempenho ocupacional e satisfação quando comparados antes e após um programa de intervenção de Terapia Ocupacional. Os resultados vão ao encontro dos estudos anteriores que demonstram o benefício da intervenção de Terapia Ocupacional com pacientes pós-AVE (LEGG, 2003; LEGG; DRUMMOND; LANGHORNE, 2006; STEULTJENS et al., 2003; WALKER, 1999).

Todavia, algumas questóes precisam ser discutidas a fim de esclarecer aspectos que podem ter influenciado nos resultados.

O uso da abordagem centrada no cliente permite parceria entre terapeuta e paciente, a distribuição de poder envolvida nessa relação; uma relação onde terapeuta se coloca como um elemento facilitador e o uso de um modelo mais preocupado com a habilitação segundo metas significativas para o cliente (LAW et al.,2009; MÂNGIA, 1999). Estudos demonstram a associação da prática centrada no cliente com a maior satisfação e adesão do 
cliente aos serviços de saúde, comparada a outros modelos de prática (DEDDING et al., 2004; WRESSLE et al., 2002) e que, quando a escolha do cliente e a autoavaliação são incorporadas ao tratamento, há aumento da motivação e participação do cliente favorecendo sua recuperação funcional (CUP et al., 2003).

Sabe-se também que o tempo de início do tratamento influencia no potencial de melhora do paciente, de forma que quanto mais precoce se inicia o processo de reabilitação maior o potencial de melhora (CRUZ, 2012). Estudo de Gilbertson et al. (2000) mostrou melhora no resultado de desempenho e satisfação de pacientes pós-AVE após programa de Terapia Ocupacional desenvolvido imediatamente após a alta hospitalar.

Os benefícios de programas de reabilitação 1 ano pós-AVE apresentam evidências inconclusivas (WALKER; SUNNERHAGEN; FISHER, 2013). Porém, vários fatores socioeconômicos, como falta de suporte econômico e familiar por parte do paciente, o número reduzido de profissionais terapeutas ocupacionais e de vagas nos serviços, influenciam e dificultam o acesso precoce dos pacientes no Brasil aos programas de reabilitação, especialmente aos de Terapia Ocupacional. Dessa forma, a diferença entre o tempo de início do tratamento, que variou de 6 a 31 meses, e a diferença no número de atendimentos semanais, que variou de 1 a 2 vezes na semana, podem ter influenciado os resultados. O estudo sugere que intervençôes de Terapia Ocupacional, mesmo com início tardio, podem trazer benefícios para o paciente, aumentando seu desempenho e satisfação.

\section{Considerações finais}

Através deste estudo foi possível verificar a melhora da percepção de pacientes pós-AVE em relação à satisfação e ao desempenho ocupacional em atividades autolistadas como importantes e significativas após intervenção de Terapia Ocupacional. Contudo, estudos futuros com grupo controle e com amostra maior e mais homogênea são sugeridos para confirmar a melhora no desempenho e satisfação dos pacientes pós-AVE após acompanhamento terapêutico ocupacional.

\section{Referências}

CUP, E. H. et al. Reliability and validity of the canadian occupational performance measure in stroke patients. Clinical Rehabilitation, London, v. 17, n. 4, p. 402-409, 2003. http://dx.doi. org/10.1191/0269215503cr635oa
CRUZ, D. M. C. Terapia Ocupacional na reabilitação pós-acidente vascular encefálico : atividades de vida diária e interdisciplinaridade. São Paulo: Santos, 2012.

DEDDING, C. et al. Validity of the canadian occupational performance measure: a client-centred outcome measurement. Clinical Rehabilitation, London, v. 18, n. 6, p. 660-667, 2004. http://dx.doi. org/10.1191/0269215504cr746oa

DIGGLE, P. J.; LIANG, K-Y.; ZEAGER, S. L. Analysis of longitudinal data. Oxford: Oxford University Press, 2002.

DOBKIN, B. H. Strategies for stroke rehabilitation. The Lancet: Neurology, London, v. 3, n. 9, p. 528-536, 2004.

DRUMOND, A. F. Fundamentos de Terapia Ocupacional. In: CAVALCANTI, A.; GALVÃO, C. Terapia Ocupacional: fundamentação e prática. Rio de Janeiro: Guanabara Koogan, 2007. cap. 2. p. 10-17.

FISHER, R. J. et al. The implementation of evidencebased rehabilitation services for stroke survivors living in the community: the results of a Delphi consensus process. Clinical Rehabilitation, London, v. 27, n. 8, p. 741-749, 2013. PMid:23405023. http://dx.doi. org/10.1177/0269215512473312

GILBERTSON, L. et al. Domiciliary occupational therapy for patients with stroke discharged from hospital: randomised controlled trial. British Medical Journal, London, v. 320, n. 4, p. 603-606, 2000. http://dx.doi. org/10.1136/bmj.320.7235.603

GOSMAN-HEDSTROM, G.; CLAESSON, L.; BLOMSTRAND, C. Assistive devices in elderly people after stroke: a longitudinal, randomized study. The Göteborg 70 + Stroke Study. Scandinavian Journal of Occupational Therapy, Oslo, v. 9, n. 3, p. 109-118, 2002. http://dx.doi.org/10.1080/11038120260246941

HOSSNE, W. S. Metodologia cientifica: para a área da saúde. São Paulo: UNICAMP, 1984.

LAVADOS, P. M. et al. Stroke epidemiology, prevention, and management strategies at a regional level: Latin America and the Caribbean. The Lancet: Neurology, London, v. 6, n. 4, p. 362-372, 2007.

LAW, M. et al. Medida Canadense de Desempenho Ocupacional (COPM). Organização e Tradução de Ana Amélia Cardoso, Lílian V. Magalhães e Lívia de C. Magalhães. Belo Horizonte: Ed. UFMG, 2009.

LEGG, L. A. Therapy-based rehabilitation services for stroke patients at home. Cochrane Database of Systematic Reviews, Oxford, n. 3, 2003. (Art. no. CD002925).

LEGG, L. A.; DRUMMOND, A. E.; LANGHORNE, P. Occupational therapy for patients with problems in activities of daily living after stroke. Cochrane Database of Systematic Reviews, Oxford, n. 4, 2006. (Art. no. CD003585).

LEGG, L. et al. Occupational therapy for patients with problems in personal activities of daily living after stroke: systematic review of randomised trials. British Medical Journal, London, v. 335, n. 7626, p. 922, 2007. PMid:17901469 PMCid:PMC2048861. http://dx.doi. org/10.1136/bmj.39343.466863.55 
MÂNGIA, E. F. Terapia ocupacional: práticas, discursos e a questão da legitimidade científica. Revista de Terapia Ocupacional da USP, São Paulo, v. 10, n. 2-3, p. 60-68, 1999.

PEDRETTI, L. W.; EARLY, M. B. Desempenho ocupacional e modelos de prática para disfunção física. In: PEDRETTY, L. W.; EARLY, M. B. Terapia Ocupacional: capacidades práticas para disfunçóes físicas. São Paulo: Roca, 2004. cap. 1. p. 10-11.

STEULTJENS, E. M. J. et al. Occupational Therapy for stroke patients: a systematic review. Stroke, Dallas, v. 34, p. 676-687, 2003. PMid:12624291. http://dx.doi. org/10.1161/01.STR.0000057576.77308.30
WALKER, M. F.; SUNNERHAGEN, K. S.; FISHER, R. J. Evidence-based community stroke rehabilitation. Stroke, Dallas, v. 44, p. 293-297, 2013. PMid:23093614. http://dx.doi.org/10.1161/STROKEAHA.111.639914

WALKER, M. F. et al. Occupational therapy for stroke patients not admitted to hospital: a randomized controlled trial. Lancet, London, v. 354, n. 9175, p. 278-280, 1999. http://dx.doi.org/10.1016/S0140-6736(98)11128-5

WRESSLE, E. et al. Improved client participation in the rehabilitation process using a client-centred goal formulation structure. Journal of Rehabilitation Medicine, Sweden, v. 34, n. 1, p. 5-11, 2002. http:// dx.doi.org/10.1080/165019702317242640

\section{Colaboração dos Autores}

Kátia Vanessa Pinto de Meneses foi responsável pela análise dos dados e redação do texto. Jeanine Schuabb Duarte, Vanessa de Oliveira Alencar e Ana Carolina dos Santos Pereira foram responsáveis pela coleta de dados e redação do texto. Todas as autoras aprovaram a versão final do texto. 